\title{
INITIAL ENERGY AND PERIHELION DISTRIBUTIONS OF OORT-CLOUD COMETS
}

\author{
P. R. WEISSMAN
}

\begin{abstract}
A Monte Carlo model of stellar perturbations of the Oort cloud is used to study the distributions in energy and perihelion of comets entering the planetary region for the first time. The model is run for a variety of initial states and a range of velocity perturbations. In all cases the resulting orbits are uniformly distributed in perihelion distance in the planetary region, $q<20 \mathrm{AU}$. Most orbits are confined to a fairly narrow range in $1 / a$ and huperbolic orbits are rare.
\end{abstract}

The observed distribution of perihelia of long-period comets, peaking near one astronomical unit, is strongly influenced by observational selection. An attempt to account for observational factors hy Everhart (1967) was successful at indicating what the intrinsic distribution looks like at small perihelion distances. But the poor statistics for comets with perihelia greater than about two $\mathrm{AU}$ as well as their low discovery probability, resulted in high uncertainty in the derived distribution at those distances.

An alternative approach to the problem is to consider a simple dynamic model of the Oort cloud and then find what perihelion distribution would result from that model. The energy distribution can also he found. This has heen done using a Monte Carlo method to duplicate the random scattering of comets from the Oort cloud into the planetary region by stellar perturbations.

The model assumes that comets are ejected to the Oort cloud on some initial orbit defined by perihelion distance $q_{0}$ and aphelion distance $Q$, randomly oriented in space. While in the cloud the comets receive repeated, randomly directed perturbations from passing stars and interstellar clouds. The sum of these perturbations may be statistically treated as a single $\Delta V$ applied to each comet's aphelion velocity vector, randomly oriented and distributed in magnitude according to a Maxwell velocity distribution characterized by root-mean-square velocity $\Delta V_{0}$. The energy and perihelia of the perturbed orhits can then be calculated and by running many cases, statistics of the resulting distributions can be found.

This model is in agreement with the results of Wyatt and Faintich (1971) who found that the Oort cloud had been "thermalized" beyond $10^{4}$ All from the sun with a rms velocity of 120 meters/second. An independent, unpublished calculation by the author found a rms velocity of $119 \mathrm{~m} / \mathrm{s}$. These velocities are 
comparable to the circular orbit velocity at a distance of $5 \times 10^{4}$ AU from the sun, the approximate aphelion distance of comets entering the solar system from the Dort sloud for the first time found by Marsden and Sekanina (1973). The summed stellar perturhations are thus capable of radically changing a comet's initial orbit even to the point of ejecting the comet from the sun's gravity field.

The computer model was run for hypothetical comets with $Q=5 \times 10^{4}$ AU. The actual distance is unimportant since the results can be scaled to any reasonable value for $Q$. Fifty cases were run encompassing five possible initial perihelion distances: $q_{0}=30,100,10^{3}, 10^{4}$, and $5 \times 10^{4}$ AU; and ten values of $\Delta V_{0}$ evenly spaced from 20 to $200 \mathrm{~m} / \mathrm{s}$. It was felt that these fifty cases covered all reasonable possibilities.

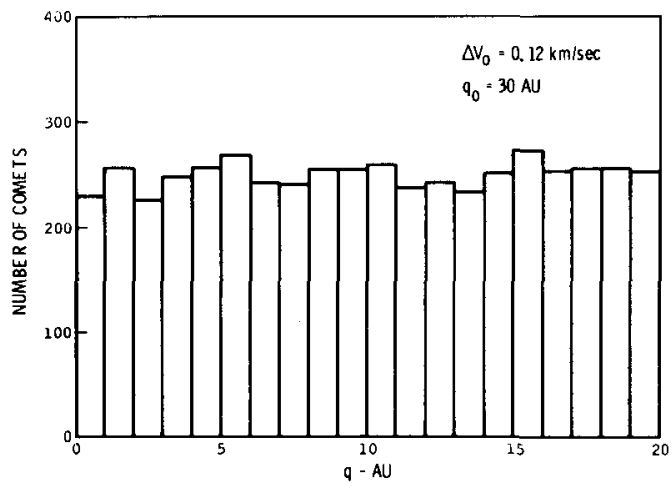

Figure 1. Perihelion distribution of oort cloud comets entering the solar system.

Results for a typical case with $q_{0}=30$ All and $\Delta V_{0}=120 \mathrm{~m} / \mathrm{s}$ are shown in Figures 1-4. Figure 1 shows the perihelion distribution hetween zero and 20 All for 5000 hypothetical comets entering the planetary region from the Oort cloud. The distribution is uniform within the limits of statistical variation. The identical result was obtained for all 50 cases. This is a result of the relatively slowly varying nature of the perturbing velocity function over the small range of aphelion velocities that can result in periholia in the solar system.

This important result is not in agreement with Everhart's derived distribution at perihelia less than one AU which showed the number of comets increasjng with $q$. However Everhart did not differentiate hetween "new" Oort cluud comets. and "old" comets which had evolved hoth physically and dynamically. It is fairly reasonable to assume that comets at small perihelion distances are more rapidly devolatized and disappear from the solar system sooner than their large perihelion counterparts. Also, planetary and non-gravitational perturbations are typically greatest for comets with small perihelion distances and may lead to a more rapid dynamical ejection from the solar system or evolution to short-period orbit.

Figure 2 shows the relative energy distribution of the same 5000 comet sample. The distribution is sharply peaked with $84.8 \%$ of all comets falling between $1 / \mathrm{a}$ values of 27 and $40 \times 10^{-6} \mathrm{AU}^{-1}$. Only $1.4 \%$ of the comets entered the planetary region on hyperbolic orbits. This, however, is an artifact of the computer model which assumes only a single perturbation at a single point in space. In a more detailed model virtually no hyperbolic orbits entering the solar system would be seen. For the same reason the lack of comets at values of 1/a greater than $40 \times 10^{-6} \mathrm{AU}^{-1}$ is artificial, and some comets at smaller 


\section{DISTRIBUTION OF OORT-CLOUD COMETS}

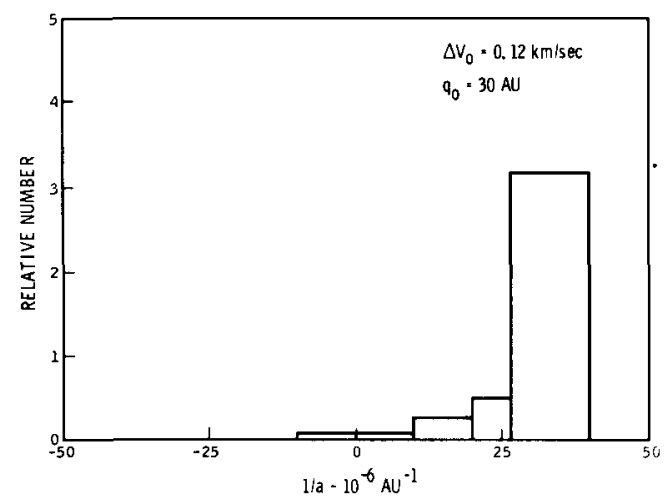

Eigure 2. Energy distribution of oort cloud comets entering the solar system.

semi-major axes would he expected. However the overall features of the distribution are essentially correct.

For the full 50 cases studied it was found that the energy distribution was independent of the initial orbit, and only a function of $\Delta V_{0}$. For small values of $\Delta V_{O}$ the distribution was sharply peaked near $40 \times 10^{-6} \mathrm{AU}^{-1}$, and as $\Delta V_{o}$ increased the distribution became more spread towards smaller values of $1 / a$.

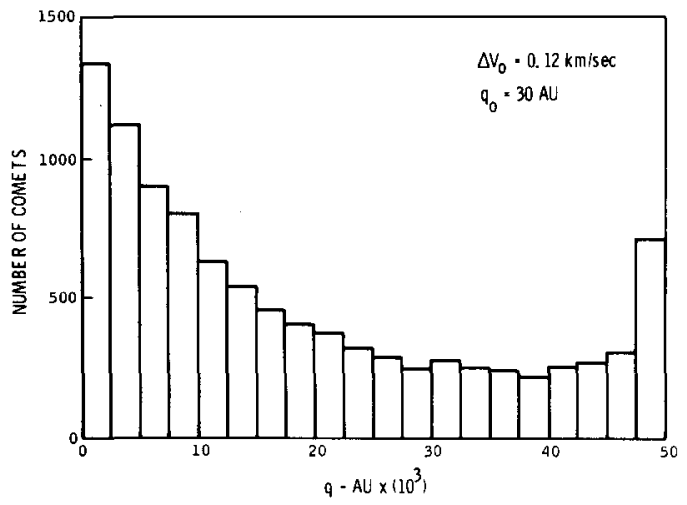

Figure 3. Perihelion distribution of comets in the oort cloud.

Figures 3 and 4 are similar distributions in perihelion and energy for the entire Oort cloud, each based on $10^{4}$ hypothetical comets. The distribution of perihelia is different for each of the 50 cases studied depending on the values of $q_{0}$ and $\Delta V_{0}$. Note in Figure 3 that the comets, originally all with $q_{0}=30 \mathrm{AU}$, have now been spread in perihelion distance throughout the cloud. The apparent increase of perihelia near $5 \times 10^{4}$ AlJ is again an artifact of the model which does not allow perihelia greater than $5 \times 10^{4}$ AU.

The energy distribution for the cloud is different in each case, reflecting both the energy of the initial orbit and the perturbing velocity. Again, statistics for hyperbolic comets are incorrect since these comets would auickly be lost from the cloud into interstellar space. Study of the fifty cases has shown that as the values of both $q_{0}$ and $\Delta v_{0}$ increase, the number of escapers from the 


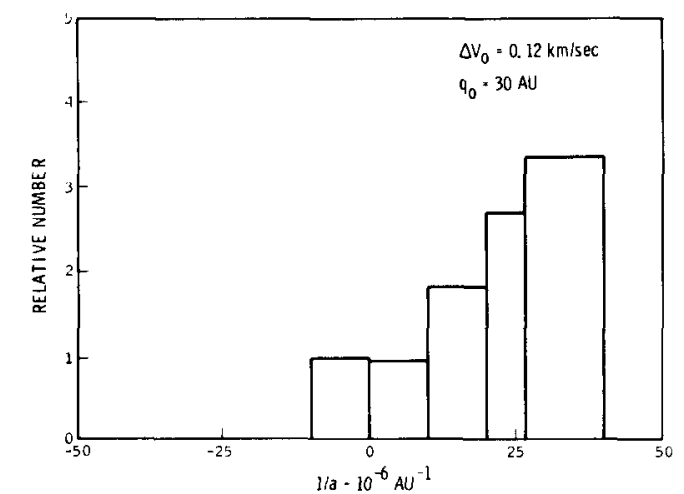

Figure 4. Energy distribution of comets in the Dort cloud.

Dort cloud increases. These results are expected to be of value in future studies of depletion of the cloud due to stellar perturbations, and the rate at which Oort cloud comets will diffuse into orbits with perihelia in the planetary region.

Returning to Figures 1 and 2 one can ask if it is possible to compare these histograms with the observed distributions to learn anything about dynamical processes in the Oort cloud. Because all cases give the same uniform perihelion distribution there is no way to discern any information from the ohserved perihelia. Since the energy distribution does reflect the magnitude of the velocity perturbation, the observed $1 /$ a distribution would be a clue to the value of $\Delta V_{0}$. However at present hoth the number and accuracy of known original orbits of long-period comets is too low to be able to draw any conclusions on this point.

\section{ACKNOWLEDGEMENTS}

The author wishes to thank William Kaula who suggested the method for investigating this problem. This work was supported by NASA grant NGL 05-0107-012.

\section{REFERENCES}

Everhart, E. 1967, Astron. J., 72, 1002.

Marsden, B. G., and Sekanina, 2. 1973, Astron, J., 78, 1118.

Wyatt, S. P., and Faintich, M. B. 1971, B.A.A.S., 3, 368.

\section{DISCUSSION}

DONN: Does your initial distribution assume all comets have the same value of $q=q_{0}$ ? This is an arbitrary severe restriction. A continuous distribution of $q$ seems more reasonable. What would be the effect of having a distribution of $q ?$

WEISSMAN: A continuous $q$ distribution is certainly reasonable. But since all values of $q_{0}$ will result in a uniform peribelion distribution, one can simply sum the possible $q$ values one wishes to consider, and will still arrive at a uniform $g$ distribution in the planetary region.

VAN FLANDERN: "New" comets show the same defect of low peribelion distances as 


\section{DISTRIBUTION OF OORT-CLOUD COMETS}

for long-period comets. How can an oort cloud origin for these he supported, in view of your result that the distribution must be flat?

WEISSMAN: New comets do not show the same defect at small perihelion distances. If one repeats the Everhart calculation using only comets which appear to be dynamically new, the statistics are too poor to say anything definite about the shape of the distribution. Of Everhart's 256 comet sample only about 49 can be identified as probably of Oort cloud origin and the sample is simply too small. One must be careful here to distinguish between the observed distribution which does drop off at small as well as very large perihelia, and the corrected distribution using Everhart's observational selection criteria. 